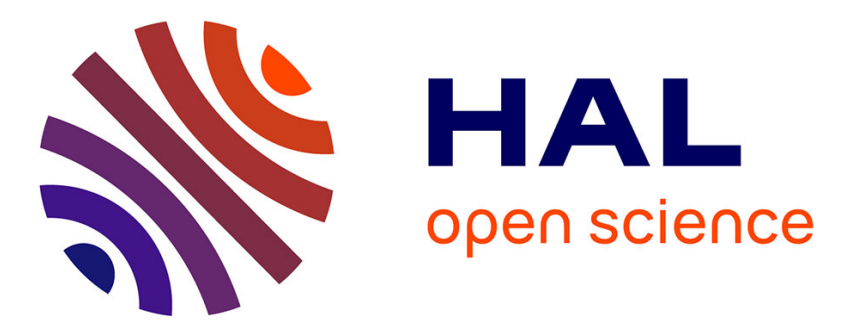

\title{
Dependence of DNA Persistence Length on Ionic Strength and Ion Type
}

Sébastien Guilbaud, Laurence Salomé, Nicolas Destainville, Manoel Manghi, Catherine Tardin

\section{- To cite this version:}

Sébastien Guilbaud, Laurence Salomé, Nicolas Destainville, Manoel Manghi, Catherine Tardin. Dependence of DNA Persistence Length on Ionic Strength and Ion Type. Physical Review Letters, 2019, 122 (2), pp.028102. 10.1103/PhysRevLett.122.028102 . hal-02024889

\section{HAL Id: hal-02024889 \\ https://hal.science/hal-02024889}

Submitted on 4 Jul 2019

HAL is a multi-disciplinary open access archive for the deposit and dissemination of scientific research documents, whether they are published or not. The documents may come from teaching and research institutions in France or abroad, or from public or private research centers.
L'archive ouverte pluridisciplinaire HAL, est destinée au dépôt et à la diffusion de documents scientifiques de niveau recherche, publiés ou non, émanant des établissements d'enseignement et de recherche français ou étrangers, des laboratoires publics ou privés. 


\title{
Dependence of DNA Persistence Length on Ionic Strength and Ion Type
}

\author{
Sébastien Guilbaud, ${ }^{1, \$}$ Laurence Salomé, ${ }^{1}$ Nicolas Destainville, ${ }^{2}$ Manoel Manghi, ${ }^{2, *}$ and Catherine Tardin ${ }^{1, \dagger}$ \\ ${ }^{1}$ Institut de Pharmacologie et de Biologie Structurale, Université de Toulouse, CNRS, UPS, 31077 Toulouse, France \\ ${ }^{2}$ Laboratoire de Physique Théorique (IRSAMC), Université de Toulouse, CNRS, UPS, 31062 Toulouse, France
}

(Received 28 September 2018; published 18 January 2019)

\begin{abstract}
Even though the persistence length $L_{P}$ of double-stranded DNA plays a pivotal role in cell biology and nanotechnologies, its dependence on ionic strength $I$ lacks a consensual description. Using a highthroughput single-molecule technique and statistical physics modeling, we measure $L_{P}$ in the presence of monovalent $\left(\mathrm{Li}^{+}, \mathrm{Na}^{+}, \mathrm{K}^{+}\right)$and divalent $\left(\mathrm{Mg}^{2+}, \mathrm{Ca}^{2+}\right)$ metallic and alkyl ammonium ions, over a large range $0.5 \mathrm{mM} \leq I \leq 5 \mathrm{M}$. We show that linear Debye-Hückel-type theories do not describe even part of these data. By contrast, the Netz-Orland and Trizac-Shen formulas, two approximate theories including nonlinear electrostatic effects and the finite DNA radius, fit our data with divalent and monovalent ions, respectively, over the whole $I$ range. Furthermore, the metallic ion type does not influence $L_{P}(I)$, in contrast to alkyl ammonium monovalent ions at high $I$.
\end{abstract}

DOI: 10.1103/PhysRevLett.122.028102

The experimental and theoretical study of polyelectrolyte stiffness has been an active field of research in the past 40 years [1-8] because its potential implications in biology, biophysics, and biotechnologies are tremendous. The diverse ionic conditions existing in the intracellular surroundings, in terms of both ionic strength and ion species [9-11], impact most of the biological macromolecules, particularly the double-stranded DNA (dsDNA), which bears one of the highest negative linear density of charges among biopolymers ( $2 e^{-}$per base pair). In nanotechnological applications, salt conditions determine the capacity of self-assembling of single-stranded DNA as well as the mechanical properties of the resulting nanostructures [12,13], e.g., DNA origami [14-17] or aptamers [18]. Even though various fields of science are concerned, how ionic conditions influence dsDNA stiffness remains controversial from a physical perspective. Stiffness is quantified by the bending persistence length $L_{P}$, the tangent-tangent correlation length, which has two contributions: a bare one, $L_{P}^{0}=K /\left(k_{B} T\right)$, related to the bending modulus $K$ [19], and an electrostatic one associated with electrostatic repulsion within the polyelectrolyte, which is partially screened as its surrounding is enriched in counterions. As a result, $L_{P}$ decreases when ion concentration grows; however, strong discrepancies exist between various experimental results obtained with different techniques [20,21], notably in force-free conditions [20] or in stretching experiments [7,22]. Furthermore, as discussed below, theoretical approaches struggle with providing a consensual frame embracing the whole range of ionic conditions.

Accurate experimental quantifications of these $L_{P}$ changes are indeed nontrivial as the data analysis is not usually straightforward [20]. Recently, we have established a methodology based on high-throughput tethered particle motion [HTTPM, see Fig. 1(a)], in which a high density of individual dsDNA molecules are tethered to a micropatterned surface by one of their extremities, while the other one is labeled with a submicrometer-sized nanoparticle [23]. Tracking the nanoparticles thus allows us the monitoring of the conformational dynamics of single dsDNA in almost force-free conditions [24]. Following a procedure of analysis based on statistical physics modeling, we established a rigorous method to retrieve $L_{P}$ from the rms of the projected end-to-end distance of the tethered particles, $R_{\text {expt| }}$ [25], and quantify its decrease as a function of the ionic strength, $I=\frac{1}{2} \sum_{i} z_{i}^{2} c_{i}$, with $z_{i}$ the valence (in units of the elementary charge $e$ ) and $c_{i}$ the concentration of ion $i$ (see Fig. 1).

From a theoretical perspective, the popular OdijkSkolnick-Fixman (OSF) model $[1,2]$ assimilates the polyelectrolyte to an infinitely thin and rigid rod with a uniform linear density of charges $A^{-1}$ ( $=6 e / \mathrm{nm}$ for dsDNA where $e$ is the elementary charge). The mobile ions, regarded as pointlike, organize in space according to the Boltzmann distribution, where the electrostatic potential is determined by linearizing the mean-field Poisson-Boltzmann (PB) equation, in the Debye-Hückel approximation valid for low electrostatic potentials. The OSF theory leads to

$$
L_{P}=L_{P}^{0}+\frac{\ell_{B}}{4 A^{2} \kappa^{2}},
$$

where $\kappa=\left(8 \pi \ell_{B} I\right)^{1 / 2}$ is the Debye parameter, and $\ell_{B}=$ $e^{2} /\left(4 \pi \varepsilon k_{B} T\right) \simeq 0.7 \mathrm{~nm}$ at $20^{\circ} \mathrm{C}$ in water is the Bjerrum length. Because of the hypothesis of low electrostatic potential, OSF theory is only valid for high $I$, typically above $0.1 \mathrm{M}$. At low $I$, a correction to the OSF model was proposed by Manning [3], where part of the ions condense 

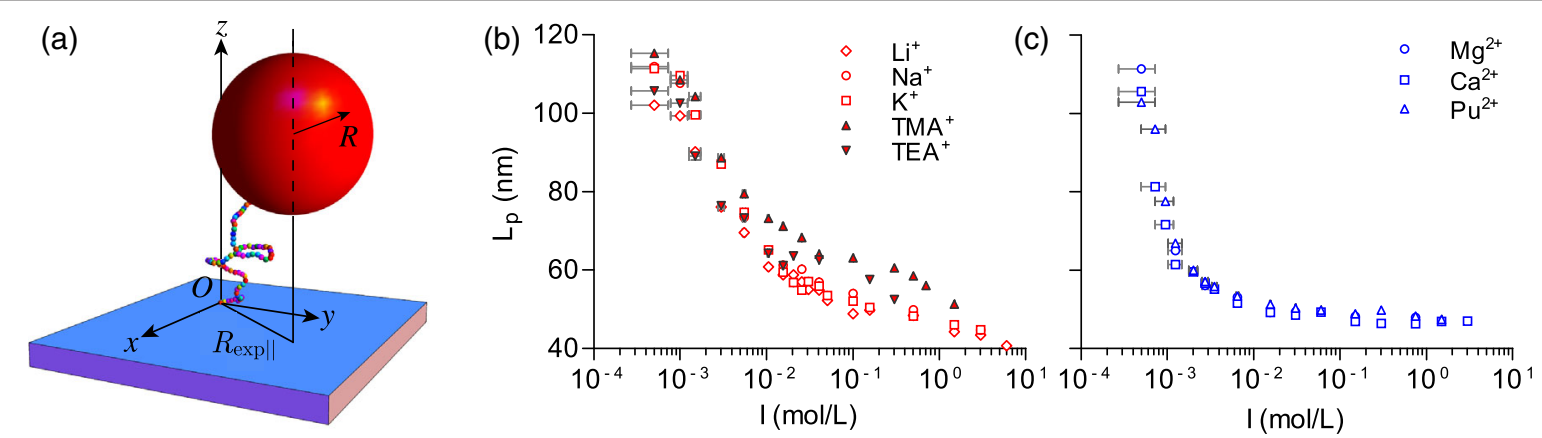

FIG. 1. (a) Sketch of the tethered particle motion setup. The measured quantity is $R_{\text {expt|| }}$. Right: Influence of the ionic strength on the mean persistence length of a 1201-bp DNA for (b) monovalent and (c) divalent cations. Error bars are displayed, if not, they are smaller than the symbol size, including vertical bars (see SM Table IV [26]).

along the DNA so that the distance between the unscreened DNA elementary charges increases up to $z \ell_{B}$. The resulting OSF-Manning (OSFM) model leads to

$$
L_{P}(I)=L_{P}^{0}+\alpha^{2}(z) \frac{\ell_{B}}{4 A^{2} \kappa^{2}},
$$

where the effective fraction of charges along the DNA $\alpha=A /\left(z \ell_{B}\right)$ depends on $z$. In order to embrace the whole range of $I$ explored experimentally, a model developed by Netz and Orland (NO) [34] and adapted in Ref. [20] is based on a variational approximation of the full PB equation. This NO theory leads to a more complicated effective charge $\alpha\left(z, \kappa R_{\mathrm{DNA}}\right)$ that depends on the DNA radius $R_{\mathrm{DNA}}$ and grows with $I$. Finally, in 2016, Trizac and Shen (TS) corrected the OSFM formula by taking into account the first term in an expansion in $\kappa R_{\mathrm{DNA}}$ of the electrostatic potential, and interpolating between exact solutions of the PB equation (in the limits of zero and high salt) for the effective charge of the DNA $\xi_{\text {eff }}$, that also varies with $\kappa R_{\mathrm{DNA}}$ [35]. Valid only for monovalent ions, it yields the same form as Eq. (2) with $\alpha$ replaced by $\alpha=\left[\left(A \xi_{\text {eff }}\right) / \ell_{B}\right]\left(1+\kappa R_{\text {DNA }}\right)^{1 / 2}$. Hence, the TS formula differs from the NO one by the corrective term and the expression of the effective charge (computed variationally in the NO approach).

In Ref. [20], data were obtained following this HTTPM procedure with $\mathrm{Na}^{+}$and $\mathrm{Mg}^{2+}$ ranging from $10 \mathrm{mM}$ to $3 \mathrm{M}$ and $0.3 \mathrm{M}$, respectively. The first three models were used to fit the data. The OSF and OSFM models could not account quantitatively for the whole experimental data set obtained with $\mathrm{Na}^{+}$or $\mathrm{Mg}^{2+}$. For the range of $I$ studied, a reasonable scaling interpolation of the NO factor was $\alpha \propto\left(\kappa R_{\mathrm{DNA}}\right)^{\beta(z)}$, where $\beta(z)$ is an effective exponent. The NO approach could then fit the $\mathrm{Mg}^{2+}$ data only, while the Manning stretching model [36], which incorporates the internal stretching of the polymer modified by ion screening, succeeded in fitting the $\mathrm{Na}^{+}$data only.

In this Letter, challenging further the existing theories predicting $L_{P}(I)$, we examine a 1201-bp dsDNA [26] (i) on an extended range of $I$ down to $0.5 \mathrm{mM}$ and up to $6 \mathrm{M}$ (under well-controlled $p \mathrm{H}$ comprised between 7 and 7.3) and (ii) with a variety of ions with different ion-specific characteristics (such as radius or hydrophobicity), neglected in all the existing theories [37] (see Fig. 1). We took much care to evaluate the influence of a large set of biologically and biotechnologically relevant ions: $\mathrm{Li}^{+}, \mathrm{Na}^{+}, \mathrm{K}^{+}$, tetramethyl ammonium $\mathrm{TMA}^{+}$, tetraethyl ammonium $\mathrm{TEA}^{+}$, $\mathrm{Mg}^{2+}, \mathrm{Ca}^{2+}$, putrescine $\mathrm{Put}^{2+}$ [see Supplemental Material (SM), Table I [26] ]. We confirm that neither the OSF theory nor its Manning refinement (OSFM) describe even part of the data. By contrast, the NO model and the TS one are shown to fit accurately the data obtained with the chosen divalent and monovalent ions, respectively, and up to $I=1 \mathrm{M}$, with reasonable values for the fitting parameters $L_{P}^{0}$ and $R_{\mathrm{DNA}}$. We therefore demonstrate in this work that the radii of metallic ions do not influence $L_{P}$ except in the case of large alkyl ammonium monovalent ions, for which a distinct $L_{P}^{0}$ at high salt is obtained.

To explore the influence of ions with this extended range of $I$ on $R_{\text {expt\| }}$, we thoroughly considered the buffer composition and the influence of $p \mathrm{H}$, which is often neglected. We became aware that, even at the usual concentration of phosphate buffer, $p \mathrm{H}$ decreased when ions were added (see SM Fig. 1 [26] and Ref. [38]). This occurred moderately for monovalent ions but quite dramatically for divalent ions (for $I>0.5 \mathrm{M}$ ). Consequently, instead of using a phosphate buffer, we chose a 4-(2hydroxyethyl)-1-piperazineethanesulfonic acid (HEPES)

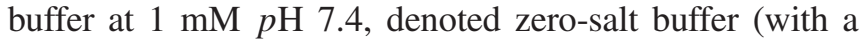
minimum ionic strength of $0.5 \mathrm{mM}$ ). Using it, $p \mathrm{H}$ is maintained between 7 and 7.3. The results obtained in the presence of $\mathrm{Na}^{+}$do not exhibit any fall at high $I$, as seen in Ref. [20]; similarly, those obtained in the presence of $\mathrm{Mg}^{2+}$ show a much less pronounced slope. This slower decrease in $R_{\text {expt } \|}$ at high $I$ is clearly correlated with the improved $p \mathrm{H}$ stabilization obtained in the 1-mM HEPES buffer, as we experimentally confirmed that acidic $p \mathrm{H}$ negatively affected $R_{\text {expt } \|}$ (see SM Fig. 1 [26]).

We then supplemented the zero-salt buffer with monovalent metallic ions $\mathrm{Li}^{+}, \mathrm{Na}^{+}, \mathrm{K}^{+}$(ionic radii ranging from 


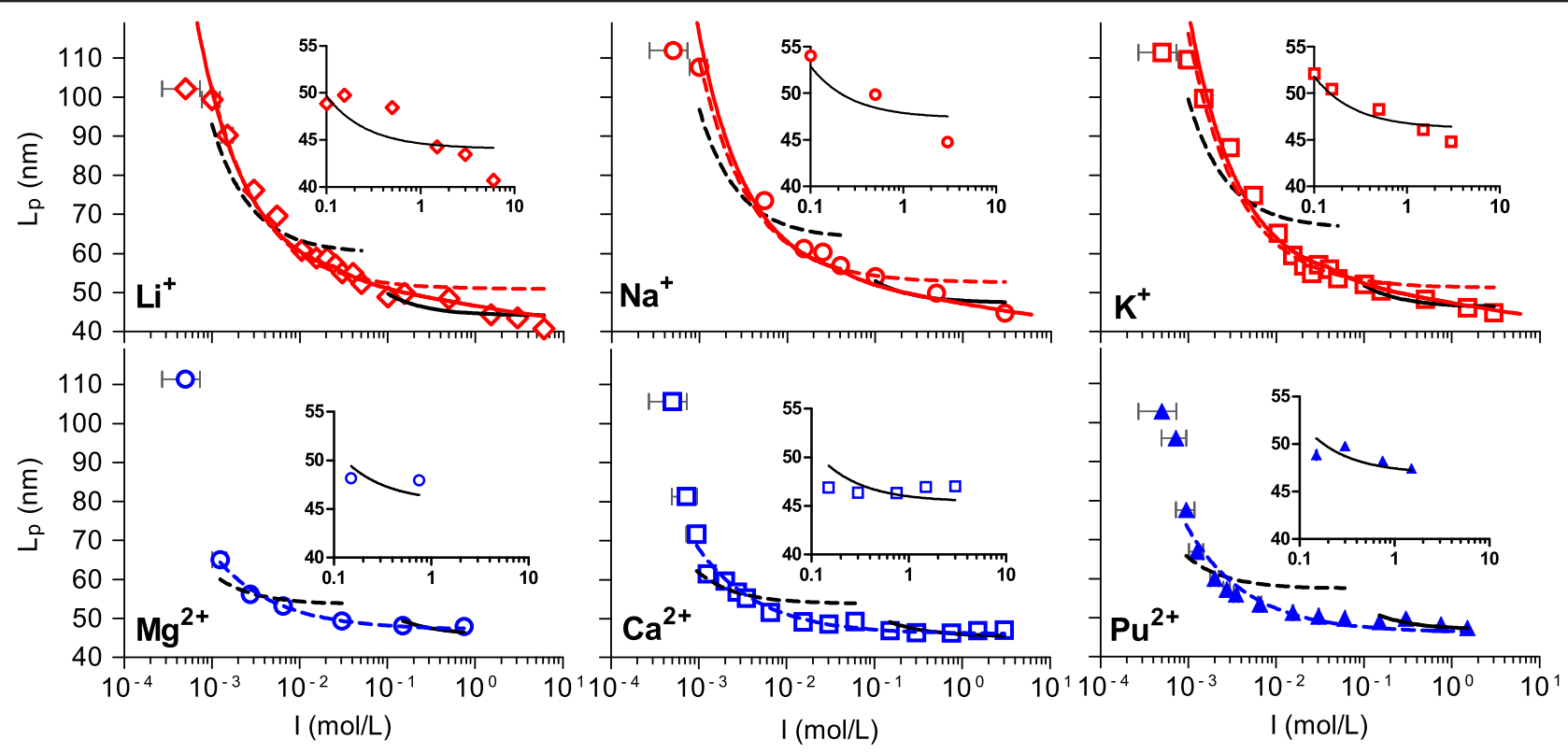

FIG. 2. Ionic strength dependence of $L_{P}$ for monovalent (top, red) and divalent (bottom, blue) ions with fitting curves corresponding to OSF $\left[L_{P}(I)=L_{P}^{0}+0.559 / I(\mathrm{~nm})\right.$, where $I$ is expressed in mol/L and $L_{P}$ in $\mathrm{nm}$; black line], OSFM (black dashed line), NO (dashed lines), and TS (solid lines) theories. Insets are enlargements of the high $I$ region. For monovalent ions, the NO model leads to $L_{P}=L_{P}^{0}+0.404 R_{\mathrm{DNA}}^{0.490} I^{-0.755}(\mathrm{~nm})\left[\right.$ with $\alpha=0.635\left(\kappa R_{\mathrm{DNA}}\right)^{0.245}$ [20] ]. For divalent ions, it leads to $L_{P}=L_{P}^{0}+0.238 R_{\mathrm{DNA}}^{0.728} I^{-0.636}$ (nm) [with $\alpha=0.423\left(\kappa R_{\mathrm{DNA}}\right)^{0.364}$ [20] ]. Error bars are displayed, if not, they are smaller than the symbol size, including vertical bars (see SM Table IV [26]).

0.071 to $0.141 \mathrm{~nm}$ ) and divalent metallic ions $\mathrm{Mg}^{2+}$ and $\mathrm{Ca}^{2+}$ (ionic radii of 0.070 and $0.103 \mathrm{~nm}$; see SM Table I [26]). When $I$ increases from $0.5 \mathrm{mM}$ to $3 \mathrm{M}, R_{\text {expt\| }}$ decreases by about $20 \%$ for both types of metallic ions (SM Fig. 2 [26]). We notice a faster decrease for divalent ions. In order to consider much larger ions, we carried out experiments with three polyamines: $\mathrm{TMA}^{+}, \mathrm{TEA}^{+}$, and $\mathrm{Put}^{2+}$ (an essential metabolite of many living organisms, e.g., found at high concentration in E. coli [39]). $\mathrm{TMA}^{+}$and $\mathrm{TEA}^{+}$ have radii 3-4 times larger than those of the chosen metallic monovalent ions. $\mathrm{Put}^{2+}$ size has not been characterized yet; however, its radius likely exceeds that of the metallic ions. Surprisingly, in the case of divalent ions, $R_{\text {expt } \|}$ values are extremely similar for both metallic ions and $\mathrm{Put}^{2+}$. In the presence of $\mathrm{TMA}^{+}$and $\mathrm{TEA}^{+}$, they are significantly higher than those obtained with metallic monovalent ions.

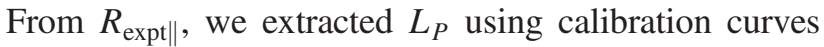
obtained by exact sampling simulation based on a statistical physics model of DNA [20]. As expected, $L_{P}$ decreases much faster when $I$ increases in the presence of divalent ions than in the presence of monovalent ions. Unexpectedly, the data superimpose on a unique curve for the three divalent ions [Fig. 1(c)] but not for the five monovalent ones [Fig. 1(b)]. In addition, $L_{P}$ reaches a plateau above $I \approx$ $50 \mathrm{mM}$ for the divalent ions while, in the case of monovalent ions, only a shoulder at $I \approx 200 \mathrm{mM}$ can be detected within the continuously decreasing curve. Molecular dynamics simulations examining the role of $\mathrm{Na}^{+}$identified a similar transitory plateau followed by a fall at high salt (SM Fig. 5
[26]) $[21,40]$. Moreover, we have measured that in the presence of monovalent ions at $I \approx 150 \mathrm{mM}$, adding even only 1-mM divalent ions leads to a significant decrease of $\approx 6 \mathrm{~nm}$ in $L_{P}$ (SM Fig. 4 [26]). This demonstrates an additive effect of monovalent and divalent ions at these biologically relevant ion concentrations.

To determine which theory best describes our experimental results, we performed fits with four equations corresponding to the OSF, OSFM, NO, and TS models. Fitting parameters are $L_{P}^{0}$, and in addition, for NO and TS models, $R_{\mathrm{DNA}}$. The fitting curves are displayed in detail in Fig. 2 and fitting parameter values and standard deviation of the residuals are given in SM Tables II and III [26]. Both OSF and OSFM formulas yield very poor fits for the whole $I$ range (see SM [26]). Since the OSF formula is theoretically valid at high salt, we fitted the data for $I \geq 100 \mathrm{mM}$. We observed discrepancies for monovalent ions, due to the absence of saturation in the experimental data. For divalent ions, the fit is correct but only for this small range of $I$. For $I \leq 100 \mathrm{mM}$, fits of $L_{P}$ using the OSFM equation were equivalently inadequate for monovalent and divalent ions. We then employed the NO model on the entire I range, excluding the very first points at low $I$ that may be partially biased due to possible plastic tube contamination (see SM section "Material and methods" [26]) but could strongly contribute to the fit due to their high $L_{P}$ value, exceeding $100 \mathrm{~nm}$. For the divalent ions, we observed extremely good adjustments of $L_{P}(I)$ by the NO fits (with $46 \leq L_{P}^{0} \leq$ $47 \mathrm{~nm}$ ) with very good values of the standard deviation of 
the residuals (see SM Table II [26]). For monovalent ions, the $\mathrm{NO}$ fits seem reasonable at low salt (for $1 \leq I \leq 30 \mathrm{mM}$ ) but systematically overestimate $L_{P}(I)$ at high salt. Moreover, the fitting values for $R_{\mathrm{DNA}}$ are small, only half the expected size of $1 \mathrm{~nm}$. To circumvent this discrepancy as well as the poor fitting at high salt, we considered the TS analytical formula [35], which considerably improved the $L_{P}(I)$ fit as observed in Fig. 2 (see SM Table III [26]). Note that the fitted value $L_{P}^{0}=41 \mathrm{~nm}$ is the same for the three metallic ions and slightly smaller than the divalent's one (fitted with a different theory), and $R_{\mathrm{DNA}}$ is almost constant, between 0.85 and $1 \mathrm{~nm}$. The strong agreement at high $I$ comes from the precise expression of $\xi_{\text {eff }}$ at large ionic strength [41]. The NO variational theory, on the other hand, looks for the optimized formula $\alpha\left(\kappa R_{\mathrm{DNA}}\right)$ for the whole $I$ range, at the expense of this high precision for large $I$, where its limiting behavior is, by construction, the OSF formula. For the $\mathrm{TMA}^{+}$and $\mathrm{TEA}^{+}$ ions the value of $L_{P}^{0}$ is slightly higher (51 and $47 \mathrm{~nm}$, respectively), which could be correlated to their large size (see SM Table I [26]).

On the basis of the TS theory, we can also explore the combined effects of the temperature $T$ and the ionic strength $I$ on $L_{P}$ on structurally intact dsDNA. In a previous work, the effect of the temperature has been measured experimentally at fixed physiological salt conditions, $I=$ $160 \mathrm{mM}$ [42]. It has been shown that $L_{P}$ decreases as $1 / T$ as expected from the simple formula valid for neutral wormlike chains $L_{P}^{0}=K /\left(k_{B} T\right)$. However, not only the bare persistence length $L_{P}^{0}$ but also the electrostatic contribution depends on $T$, since entropic effects control the ionic screening of the dsDNA. Hence, using our fitting values obtained at $T=20^{\circ} \mathrm{C}$, we plotted $L_{P}(T)$ for various $I$ in Fig. 3. We observed that for $I \geq 100 \mathrm{mM}$, the electrostatic contribution is small as compared to $L_{P}^{0}$. Therefore, we observe a $1 / T$ law with a shift of the curve to lower values when $I$ increases. For $I<4 \mathrm{mM}$, however, we predict a striking reversal with $L_{P}$ increasing with $T$.



FIG. 3. Theoretical prediction of the influence of the temperature $T$ on $L_{P}$ for monovalent ions and $I=0.001,0.004,0.01$, $0.1,1 \mathrm{M}$ from top to bottom. The dashed curve is the infinite $I$ limit showing the nonelectrostatic contribution $L_{P}^{0}=K /\left(k_{B} T\right)$.
New experiments exploring the dependence of the $L_{P}$ as a function of $T$ and $I$ are therefore needed to check further the theoretical TS approach.

No influence of the size or the nature of the ions was seen as the curves obtained with the three divalent ions and the three metallic monovalent ions superimposed in two unique curves. This complete superimposition prevents us from considering as significant the slight changes of dsDNA radius derived from the fits. The independence of the ionicstrength variation of $L_{P}$ with the ion size and our fitted values for $R_{\mathrm{DNA}} \approx 1 \mathrm{~nm}$ are in good agreement with the experimental results of Gebala and co-workers [43]. They showed that the atmosphere occupancy around dsDNA by monovalent ions did not depend on the ion size across the monovalent metallic ions except for $\mathrm{Li}^{+}$[43], which only led to a slight reduction as compared to the other monovalent metallic ions. These two distinct approaches thus support the same view of an identical behavior for various metallic monovalent ions with different sizes. Therefore, in timescales of seconds, the differences in the ion binding sites along the DNA tube [44] and residence times [45] measured between $\mathrm{Na}^{+}$and $\mathrm{K}^{+}$using molecular dynamic simulations of tens of nanoseconds fade away.

Concerning alkyl ammonium ions, their significantly higher $L_{P}^{0}$ suggests that their 3-4 times wider ion size precludes a sufficiently large density of ions close to dsDNA to completely screen the electrostatic interactions, even at large I. The capacity of these ions to easily dehydrate and consequently enter the dsDNA grooves, as predicted by molecular dynamic simulations [46], is insufficient to screen the dsDNA charges. Hydrated divalent metallic ions such as $\mathrm{Mg}^{2+}$ were predicted to exhibit a much more localized distribution than $\mathrm{Na}^{+}$and $\mathrm{K}^{+}$and to spend long resident times of a few nanoseconds within the dsDNA tube [45]; yet, we did not measure any impact on the fitted $R_{\text {DNA }}$ value. Surprisingly, Put ${ }^{2+}$ behaves as metallic divalent ions in spite of its long linear structure. This is at odds with what was found to describe thermal DNA denaturation in the presence of $\mathrm{Put}^{2+}[39,47]$.

The great efficiency of NO and TS theories comes from the consideration of nonlinear electrostatic terms and of the finite radius of dsDNA. It suggests that they could also be valid for other polyelectrolytes. For hyaluronic acid in the presence of $\mathrm{Na}^{+}$in stretching experiments with magnetic tweezers [49], $L_{P}$ decreases following $L_{P}=L_{P}^{0}+$ const $\times$ $I^{-\delta}$ with $\delta=0.65$ at low salt to be compared with our value of 0.75 , while $L_{P}^{0}$ is about 10 times shorter than the dsDNA one, and the charge density is 6 times smaller. The use of NO and TS theories to finely model the flexibility of biopolymers such as single-stranded RNA [50] or chromatin fibers should be extremely useful for the elucidation of gene expression and 3D organization of chromosomes [51] and for the control of the shape of nucleic acid nanostructures [52].

We acknowledge Philippe Rousseau (LMGM, CBI, Toulouse) for the DNA samples. 
*manghi@irsamc.ups-tlse.fr

†tardin@ipbs.fr

*Present address: Department of Physics, University of York, York YO10 5DD, United Kingdom.

[1] T. Odijk, Polyelectrolytes near the rod limit, J. Polym. Sci. 15, 477 (1977).

[2] J. Skolnick and M. Fixman, Electrostatic persistence length of a Wormlike polyelectrolyte, Macromolecules 10, 944 (1977).

[3] G.S. Manning, A procedure for extracting persistence lengths from light-scattering data on intermediate molecular weight DNA, Biopolymers 20, 1751 (1981).

[4] M. Fixman, The flexibility of polyelectrolyte molecules, J. Chem. Phys. 76, 6346 (1982).

[5] M. Le Bret, Electrostatic contribution to the persistence length of a polyelectrolyte, J. Chem. Phys. 76, 6243 (1982).

[6] J.-L. Barrat and J.-F. Joanny, Persistence length of polyelectrolyte chains, Europhys. Lett. 24, 333 (1993).

[7] C. Baumann, S. Smith, V. Bloomfield, and C. Bustamante, Ionic effects on the elasticity of single DNA molecules, Proc. Natl. Acad. Sci. U.S.A. 94, 6185 (1997).

[8] J. Wenner, M. Williams, I. Rouzina, and V. Bloomfield, Salt dependence of the elasticity and overstretching transition of single DNA molecules, Biophys. J. 82, 3160 (2002).

[9] K. Fagerbakke, M. Heldal, and S. Norland, Content of carbon, nitrogen, oxygen, sulfur and phosphorus in native aquatic and cultured bacteria, Aquatic Microbial Ecology 10, 15 (1996).

[10] M. Heldal, S. Norland, and O. Tumyr, X-Ray microanalytic method for measurement of dry matter and elemental content of individual bacteria, Appl. Environ. Microbiol. 50, 1251 (1985).

[11] B. Alberts, A. Johnson, J. Lewis, M. Raff, K. Roberts, and P. Walter, Molecular Biology of the Cell, 4th ed. (Garland Science, New York, 2002).

[12] S. H. Lee, C. K. Lee, S. R. Shin, S. I. Kim, I. So, and S. J. Kim, The peculiar response of DNA hydrogel fibers to a salt and $\mathrm{pH}$ stimulus, Macromol. Rapid Commun. 30, 430 (2009).

[13] J. D. Halverson and A. V. Tkachenko, DNA-programmed mesoscopic architecture, Phys. Rev. E 87, 062310 (2013).

[14] S. Fischer, C. Hartl, K. Frank, J. O. Rädler, T. Liedl, and B. Nickel, Shape and Interhelical spacing of DNA origami nanostructures studied by small-angle x-ray scattering, Nano Lett. 16, 4282 (2016).

[15] R. P. Goodman, I. A. Schaap, C. F. Tardin, C. M. Erben, R. M. Berry, C. F. Schmidt, and A. J. Turberfield, Rapid chiral assembly of rigid DNA building blocks for molecular nanofabrication, Science 310, 1661 (2005).

[16] A. Reinhardt and D. Frenkel, Numerical Evidence for Nucleated Self-Assembly of DNA Brick Structures, Phys. Rev. Lett. 112, 238103 (2014).

[17] F. Hong, F. Zhang, and H. Yan, DNA Origami: Scaffolds for creating higher order structures, Chem. Rev. 117, 12584 (2017).

[18] K. Padmanabhan, K. P. Padmanabhan, J. D. Ferrara, J. E. Sadler, and A. Tulinsky, The structure of alpha-thrombin inhibited by a 15-mer single-stranded DNA aptamer, J. Biol. Chem. 268, 17651 (1993).
[19] O. Kratky and G. Porod, Röntgenuntersuchung gelöster Fadenmoleküle, Recl. Trav. Chim. 68, 1106 (1949).

[20] A. Brunet, C. Tardin, L. Salomé, P. Rousseau, N. Destainville, and M. Manghi, Dependence of DNA persistence length on ionic strength of solutions with monovalent and divalent salts: A joint theory-experiment study, Macromolecules 48, 3641 (2015).

[21] A. Savelyev, Do monovalent mobile ions affect DNAs flexibility at high salt content?, Phys. Chem. Chem. Phys. 14, 2250 (2012).

[22] O. A. Saleh, D. B. McIntosh, P. Pincus, and N. Ribeck, Nonlinear Low-Force Elasticity of Single-Stranded DNA Molecules, Phys. Rev. Lett. 102, 068301 (2009).

[23] T. Plenat, C. Tardin, P. Rousseau, and L. Salomé, Highthroughput single-molecule analysis of DNA-protein interactions by tethered particle motion, Nucl. Acids Res. 40, e89 (2012).

[24] D. E. Segall, P. C. Nelson, and R. Phillips, Volume-Exclusion Effects in Tethered-Particle Experiments: Bead Size Matters, Phys. Rev. Lett. 96, 088306 (2006).

[25] M. Manghi, C.Tardin, J. Baglio, P. Rousseau, L. Salomé, and N. Destainville, Probing DNA conformational changes with high temporal resolution by tethered particle motion, Phys. Biol. 7, 046003 (2010).

[26] See Supplemental Material at http://link.aps.org/ supplemental/10.1103/PhysRevLett.122.028102 for the material and methods, experimental issues, a comparison to existing simulation results, and the fitting procedure, which includes Refs. [27-33].

[27] J. R. Rossum, Conductance method for checking accuracy of water analyses, Anal. Chem. 21, 631 (1949).

[28] S. Blumberg, A. Gajraj, M. W. Pennington, and J.-C. Meiners, Three-dimensional characterization of tethered microspheres by total internal reflection fluorescence microscopy, Biophys. J. 89, 1272 (2005).

[29] A. Brunet, S. Chevalier, N. Destainville, M. Manghi, P. Rousseau, M. Salhi, L. Salomé, and C. Tardin, Probing a label-free local bend in DNA by single molecule tethered particle motion, Nucl. Acids Res. 43, e72 (2015).

[30] Y. Marcus, Ionic radii in aqueous solutions, Chem. Rev. 88, 1475 (1988).

[31] J. M. G. Barthel, H. Krienke, and W. Kunz, Physical Chemistry of Electrolyte Solutions: Modern Aspects (Springer Science \& Business Media, New York, 1998).

[32] L. A. Yatsunyk, O. Mendoza, and J.-L. Mergny, "Nanooddities": Unusual nucleic acid assemblies for DNA-based nanostructures and nanodevices, Acc. Chem. Res. 47, 1836 (2014).

[33] P. Bevington and D. K. Robinson, Data Reduction and Error Analysis for the Physical Sciences (McGraw-Hill Education, New York, 2003).

[34] R. R. Netz and H. Orland, Variational charge renormalization in charged systems, Eur. Phys. J. E 11, 301 (2003).

[35] E. Trizac and T. Shen, Bending stiff charged polymers: The electrostatic persistence length, Europhys. Lett. 116, 18007 (2016).

[36] G. S. Manning, The contribution of transient counterion imbalances to DNA bending fluctuations, Biophys. J. 90, 3208 (2006).

[37] G. S. Manning (private communication). 
[38] C. M. H. Ferreira, I.S. S. Pinto, E. V. Soares, and H. M. V. M. Soares, (Un)suitability of the use of $p \mathrm{H}$ buffers in biological, biochemical and environmental studies and their interaction with metal ions-a review, RSC Adv. 5, 30989 (2015).

[39] L. Miller-Fleming, V. Olin-Sandoval, K. Campbell, and M. Ralser, Remaining mysteries of molecular biology: The role of polyamines in the cell, J. Mol. Biol. 427, 3389 (2015).

[40] A. Savelyev and G. A. Papoian, Chemically accurate coarse graining of double-stranded DNA, Proc. Natl. Acad. Sci. U.S.A. 107, 20340 (2010).

[41] M. Aubouy, E. Trizac, and L. Bocquet, Effective charge versus bare charge: An analytical estimate for colloids in the infinite dilution limit, J. Phys. A 36, 5835 (2003).

[42] A. Brunet, L. Salomé, P. Rousseau, N. Destainville, M. Manghi, and C. Tardin, How does temperature impact the conformation of single DNA molecules below melting temperature?, Nucl. Acids Res. 46, 2074 (2018).

[43] M. Gebala, S. Bonilla, N. Bisaria, and D. Herschlag, Does cation size affect occupancy and electrostatic screening of the nucleic acid ion atmosphere?, J. Am. Chem. Soc. 138, 10925 (2016).

[44] P. Várnai and K. Zakrzewska, DNA and its counterions: A molecular dynamics study, Nucl. Acids Res. 32, 4269 (2004).

[45] F. Pan, C. Roland, and C. Sagui, Ion distributions around left- and right-handed DNA and RNA duplexes: A comparative study, Nucl. Acids Res. 42, 13981 (2014).
[46] D. Bhowmik, N. Malikova, G. Mériguet, O. Bernard, J. Teixeira, and P. Turq, Aqueous solutions of tetraalkylammonium halides: Ion hydration, dynamics and ion-ion interactions in light of steric effects, Phys. Chem. Chem. Phys. 16, 13447 (2014).

[47] Our measurements are performed on 1201-bp-long DNA molecules for which any sequence effect $[44,48]$ is assumed to be self-averaged.

[48] S. Y. Ponomarev, K. M. Thayer, and D. L. Beveridge, Ion motions in molecular dynamics simulations on DNA, Proc. Natl. Acad. Sci. U.S.A. 101, 14771 (2004).

[49] J. P. Berezney and O.A. Saleh, Electrostatic effects on the conformation and elasticity of Hyaluronic acid, a moderately flexible polyelectrolyte, Macromol. 50, 1085 (2017).

[50] H. Chen, S. P. Meisburger, S. A. Pabit, J. L. Sutton, W. W. Webb, and L. Pollack, Ionic strength-dependent persistence lengths of single-stranded RNA and DNA, Proc. Natl. Acad. Sci. U.S.A. 109, 799 (2012).

[51] G. Zhu, W. Deng, H. Hu, R. Ma, S. Zhang, J. Yang, J. Peng, T. Kaplan, and J. Zeng, Reconstructing spatial organizations of chromosomes through manifold learning, Nucl. Acids Res. 46, e50 (2018).

[52] P. Liu, Y. Zhao, X. Liu, J. Sun, D. Xu, Y. Li, Q. Li, L. Wang, S. Yang, C. Fan et al., Charge neutralization drives the shape reconfiguration of DNA nanotubes, Angew. Chem. 57, 5418 (2018). 